\title{
PREPARATION, RELEASE, RHEOLOGY AND STABILITY OF PIROXICAM EMULGEL
}

\author{
ZAINAB T. SALIH ${ }^{1}$, FATIMA AL_GAWHARI ${ }^{2}$, NAWAL A. RAJAB ${ }^{3}$ \\ 1,2,3Department of Pharmaceutics, College of Pharmacy, University of Baghdad, Baghdad, Iraq \\ Email: dr.fatima24042011@gmail.com
}

Received: 25 May 2017, Revised and Accepted: 28 Nov 2017

\section{ABSTRACT}

Objective: The purpose of this work was to develop and optimize the emulgel formulation of piroxicam with two types of gelling agent chitosan and xanthan gum. The release profiles of prepared formulas were investigated. In addition, the rheology and stability of the best formula were investigated.

Methods: Emulsified piroxicam was prepared to use oleic acid, tween 80 and PG with a ratio (3:10:10). In xanthan based emulgel, the xanthan gum $(1 \%$ and $1.5 \%)$ was spread as powder on emulsified piroxicam with stirring until emulgel was formed. In chitosan-based emgels, Chitosan gel was added to emulsified piroxicam and stirring until the Emulgel was constructed. Chitosan gels were prepared by incorporating different concentration, $2 \%, 3 \%, 6 \% \mathrm{w} / \mathrm{v}$ of chitosan in $1 \% \mathrm{v} / \mathrm{v}$ of glacial acetic acid in distilled water. In vitro release of piroxicam from different formulas was conducted in $300 \mathrm{ml}$ phosphate buffer $\mathrm{pH} 7.4$, at a speed of $120 \mathrm{rpm}$ at $37 \pm 2^{\circ} \mathrm{C}$. The amount of the drug released from the bases was determined spectrophotometrically at $504 \mathrm{~nm}$. Viscometer Myr. Vr 3000 was used to measure the viscosity of the prepared formulas. The prepared formulas were stored in well-stoppered polyvinyl chloride (PVC) plastic containers in the dark for 6 mo at room temperature. They were checked for drug content, viscosity, and $\mathrm{pH}$ change bimonthly throughout the period.

Results: The results showed that the dissolution increases significantly with increasing the concentration of xanthan. Chitosan has significant synergized the enhancements of xanthan gum in the release. Rheological behaviour of the selected formula containing chitosan (2\%) and xanthan gum (1.5) had shear thinning in nature showing a decrease in viscosity at the increasing shear rates. The selected formula was stable 6 mo at $40^{\circ} \mathrm{C} / 75 \% \mathrm{RH}$ and $4^{\circ} \mathrm{C}$. The formula found was yellow viscous creamy preparation with the smooth homogenous appearance. The $\mathrm{pH}$ and the drug release was also found to be stable under storage conditions.

Conclusion: Piroxicam release can be improved by preparing emulgel which stable and have good rheologic properties.

Keywords: Piroxicam, Emulgel, Xanthan gum, Chitosan

(c) 2018 The Authors. Published by Innovare Academic Sciences Pvt Ltd. This is an open-access article under the CC BY license (http://creativecommons.org/licenses/by/4.0/) DOI: http://dx.doi.org/10.22159/ijap.2018v10i1.20234

\section{INTRODUCTION}

Numerous analgesic preparations are available in the pharmacy as different transdermal preparations. piroxicam causes side-effects on the gastrointestinal tract. Therefore, transdermal application of piroxicam has been used to minimize these side effects.

Predictably it is available in the form of capsules for oral administration and gel for topical use. In spite of many advantages of gels which enhance dissolution of drugs, and increases migration of the drug in comparison with the ointment or cream base a major restraint of gel is in the transfer of hydrophobic drugs e. g piroxicam and mefenamic acid. So to overcome this problem, emulgels are prepared and used so that a hydrophobic moiety can exhibit the advantages of gels [1-3]. Emulsions are the systems of two or more immiscible liquid phases. The one phase is dispersed in the dispersion medium. Emulgels consists of emulsion and gel. There are three basic steps in the preparation of emulgel. Step 1: Formulation of emulsion either $\mathrm{O} / \mathrm{W}$ or $\mathrm{W} / \mathrm{O}$. Step 2: Formulation of gel base. Step 3: Incorporation of emulsion into gel base with continuous stirring [2].

In the formulation of gel base, chitosan and natural gum (e. g. xanthan gum) can be used to get the gelling structure. Chitosan is a natural, non-toxic, biodegradable and biocompatible polymer. Gums can form a gel either alone or in combination with another polymer $[3,4]$.

The aim of this work was to develop and optimize the emulgel formulation of piroxicam with two types of gelling agent chitosan and xanthan gum. The release profiles of prepared formulas were investigated. In addition, the rheology and stability of the best formula were investigated.

\section{MATERIALS AND METHODS}

\section{Materials}

Ferric sulfate (M. W.: 399.88) was from Fluka Chemie AG, Switzerland. Piroxicam and chitosan purchased from Sigma. Chitosan was medium molecular weight (MW=103.200 g/Mol) with a $97 \%$ degree of deacetylation. Oleic acid $(\mathrm{BDH})$, xanthan gum (HIMIA), disodium hydrogen phosphate (BHD) and PEG 400 (BHD).

\section{Methods}

\section{Preparation of emulsified piroxicam}

Piroxicam was added to the mixture of oleic acid, tween80 and PG with a ratio as described in table 2 , and then an appropriate amount of water was added to the mixture drop by drop and then emulsion containing piroxicam was obtained by stirring the mixture at ambient temperature.

\section{Preparation of chitosan gel}

Chitosan gels were prepared by incorporating different concentration, $2 \%, 3 \%, 6 \% \mathrm{w} / \mathrm{v}$ of chitosan in $1 \% \mathrm{v} / \mathrm{v}$ of glacial acetic acid in distilled water. A weighted amount of chitosan was taken and dispersed over $1 \%$ glacial acetic acid overnight until all the chitosan is soaked. Then, homogenized by stirring until getting the gel structure [5, 6]. After homogenization, the formed gel was sonicated for $15 \mathrm{~min}$ to expel out the entrapped air bubbles from the prepared gel. Three chitosan-based formulations were prepared as shown in table 1.

Table 1: Compositions of chitosan based formulations

\begin{tabular}{llll}
\hline Chitosan gels formulations & Chitosan \% (w/v) & Glacial acetic acid \% v/v & Water \% w/w \\
\hline G1 & 2 & 1 & $100 \%$ \\
G2 & 3 & 1 & $100 \%$ \\
G3 & 6 & 1 & $100 \%$ \\
\hline
\end{tabular}




\section{Preparation of piroxicam emulgels}

The chitosan-based gels were added to emulsified piroxicam and stirring until the emulgel was constructed as shown in table 2. But, in xanthan based emulgel, the xanthan was spread as powder on emulsified piroxicam with stirring until emulgel was formed as shown in table 3.

Table 2: Composition of emulsion and chitosan-based emulgels formulations

\begin{tabular}{|c|c|c|c|c|c|c|}
\hline \multirow[t]{2}{*}{ Emulsion and Emulgels formulations } & \multirow[t]{2}{*}{ Piroxicam (g) } & \multicolumn{3}{|c|}{ Emulsifying agents } & \multirow{2}{*}{$\begin{array}{l}\text { Water } \\
\text { (g) }\end{array}$} & \multirow{2}{*}{$\begin{array}{l}\text { Chitosan } \\
\text { Gel (50 g) }\end{array}$} \\
\hline & & Oleic acid (g) & Tween 80 (g) & PG (g) & & \\
\hline Piroxicam emulsion & 0.5 & 3 & 10 & 10 & q. s 50 & - \\
\hline EG1 & 0.5 & 6 & 20 & 10 & q. $s 50$ & G1 \\
\hline EG2 & 0.5 & 6 & 20 & 10 & q. $s 50$ & G2 \\
\hline EG3 & 0.5 & 6 & 20 & 10 & q. s 50 & G3 \\
\hline
\end{tabular}

Table 3: Composition of emulsion and xanthan or combination of xanthan and chitosanemul gels formulations

\begin{tabular}{lllllll}
\hline Emulgels & Piroxicam (g) & \multicolumn{2}{l}{ Emulsifying agents } & \multicolumn{2}{l}{ Gelling agents } \\
\cline { 2 - 5 } formulas & & Oleic acid (g) & Tween 80 (g) & PG (g) & Xanthan gum & Chitosan \\
Q. S.
\end{tabular}

\section{Preparation of calibration curve of piroxicam}

$0.16 \%$ piroxicam $(1.6 \mathrm{mg} / \mathrm{ml})$ solution was prepared in methanol. Aliquots of $0.05-1.0 \mathrm{ml}$ standard piroxicam $(1.6 \mathrm{mg} / \mathrm{ml})$ solution corresponding to $8-160 \mu \mathrm{g} \mathrm{ml} / \mathrm{ml}$ were populated.

Into a series of $10 \mathrm{ml}$ standard volumetric flasks. To each flask, 1.0 $\mathrm{ml}$ of ferric sulfate $(0.005 \mathrm{M})$ solution was added and diluted up to the mark with ethanol. The contents of each flask were mixed well at room temperature and the absorbance was measured at $504 \mathrm{~nm}$ against the reagent blank prepared similarly except drug. The color was stable up to $24 \mathrm{~h}$. The amount of the drug was obtained from the regression equation [6].

\section{In vitro release of piroxicam from different topical formulas}

The release pattern of the drug from the formulas was examined using a dissolution apparatus, holding the temperature of the water bath at $37 \pm 2{ }^{\circ} \mathrm{C}$ (USP dissolution test apparatus II), containing 300 $\mathrm{ml}$ phosphate buffer $\mathrm{pH} 7.4$, at a speed of $120 \mathrm{rpm}$. The experimental procedure was carried out using $2 \mathrm{~g}$ of each formula. The analysis was performed with $2 \mathrm{ml}$ samples withdrawn from the dissolution medium at $15 \mathrm{~min}$ intervals. The removed samples were replaced with equal volumes of phosphate buffer at the same $\mathrm{pH}$ to maintain a constant volume of the receiving medium. The amount of the drug released from the bases was determined spectrophotometrically at $405 \mathrm{~nm}$. Experiments were done in triplicate and mean results were reported [6].

\section{Stability studies of formulas}

The prepared formulas were stored in well-stoppered polyvinyl chloride (PVC) plastic containers in the dark for 6 mo at room temperature. They were checked for drug content, viscosity, and $\mathrm{pH}$ change every two months throughout the period [6].

\section{Piroxicam content study}

An accurately weighted quantity of each formula (about $500 \mathrm{mg}$ ) was dissolved in ethanol. These solutions were quantitatively transferred to volumetric flasks and appropriate dilutions were made. The resulting solutions were then filtered through $0.45 \mu \mathrm{m}$ membrane filters before subjecting the solution to visible spectrophotometric analysis for piroxicam at $504 \mathrm{~nm}$ [6].

\section{Viscosity measurements}

Viscometer Myr. Vr 3000 was used to measure the viscosity of the prepared formulas. The spindle R6 was rotated at $10 \mathrm{rpm}$. Samples of the bases were allowed to settle over $30 \mathrm{~min}$ at room temperature before the measurements were taken.

\section{pH measurements}

The $\mathrm{pH}$ was measured in each base using a $\mathrm{pH}$ meter that was calibrated before each use with buffered solutions at $\mathrm{pH} 4,7$, and 10 . A defined amount of each tested base was taken and diluted with calibrated distilled water and mixed well. The electrode of the $\mathrm{pH}$ meter was immersed in the prepared base solution for $\mathrm{pH}$ determination.

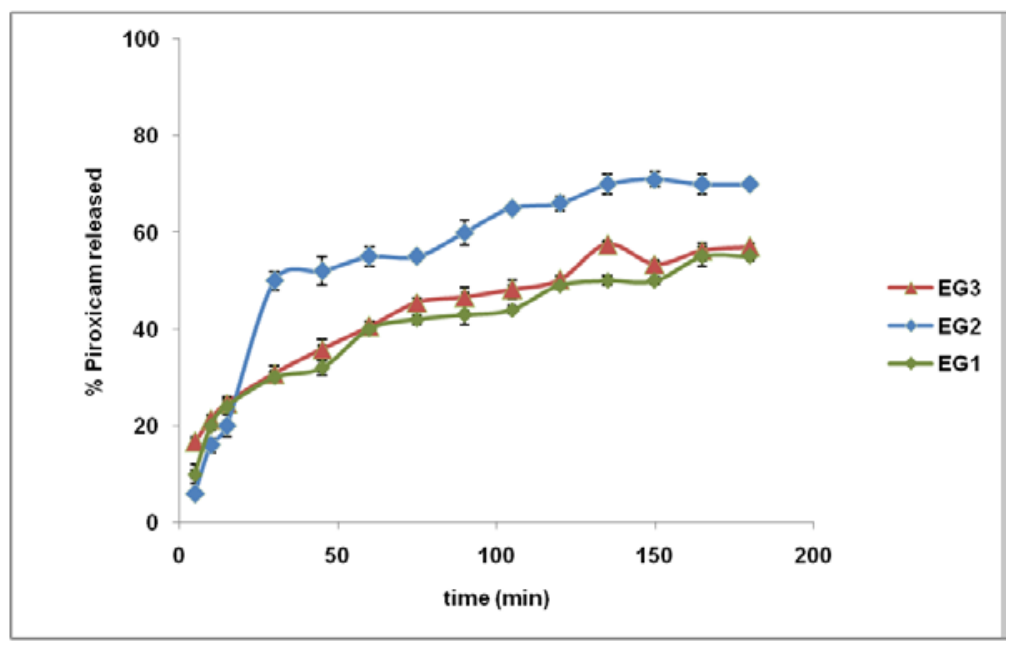

Fig. 1: The release profiles from chitosan-based emulgel, results are expressed as mean, $n=3 \pm S D$ 


\section{RESULTS AND DISCUSSION}

The results of the release profile showed that enhancement of the emulsified piroxicam release with increasing the concentration of chitosan from 1 to $2 \%(\mathrm{P}<0.05)$ due to increase the wettability of piroxicam. But, the increase in viscosity at concentration $3 \%$ cause decrease in the release of piroxicam. These may be occurred as a consequence of the increasing in the density of the polymer matrix and also an increase in diffusion layer which the drug molecules have to pass [7].

The dissolution profiles of the formulas contain xanthan gum is demonstrated in fig. 2. The results showed that the dissolution increase with increasing the concentration of xanthan $(\mathrm{P}<0.05)$. Xanthan coats the hydrophobic particles of piroxicam with a hydropillic layer, thus promotes wetting and dissolution of piroxicam [8]. Release profiles of piroxicam from the two gelling agents performs that release varies according to the type of utilizing gum or polymer. Moreover, release from both gelling agents shows synergized the enhancements $(\mathrm{P}<0.05)$. However, based on the nature of their molecular structure of each agent are expected to reveal different effects on drug release as a consequence of their dissimilar hydration, swelling, gel strength and erosion characteristics [9].

Rheological behavior of the selected formula showed that the systems were shear thinning in nature showing a decrease in viscosity at the increasing shear rates. As the shear stress is increased, the normally disarranged molecules of the xanthan gum and chitosan are beginning to align their direction. Such alignment reduces the internal resistance of the gelling agents and hence decreases the viscosity [9].

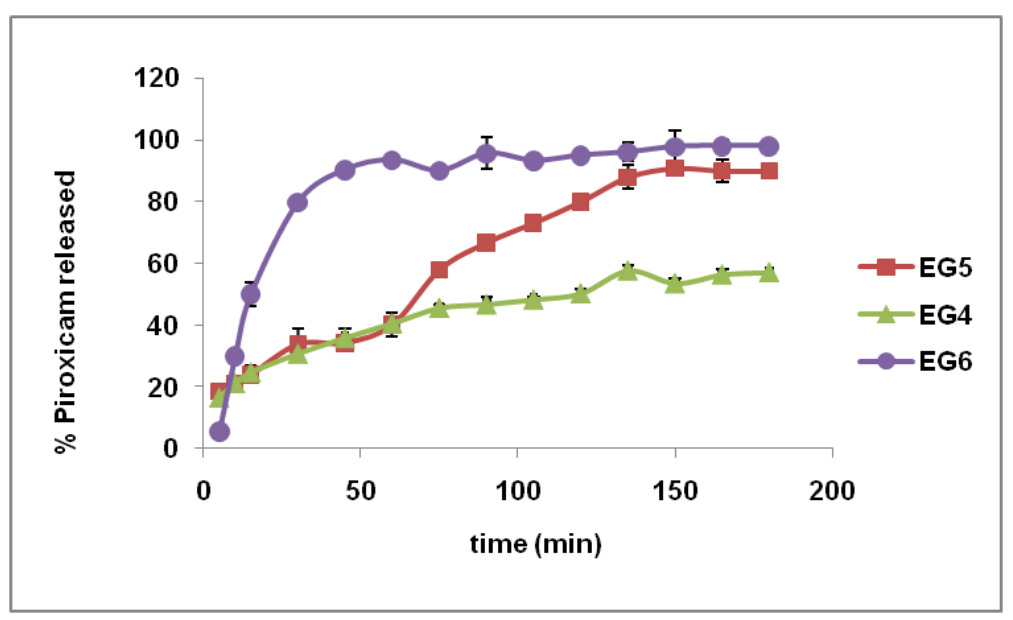

Fig. 2: The release profile from xanthan based emulgel and combined xanthan-chitosan based gel, results are expressed as mean, $n=3 \pm S D$

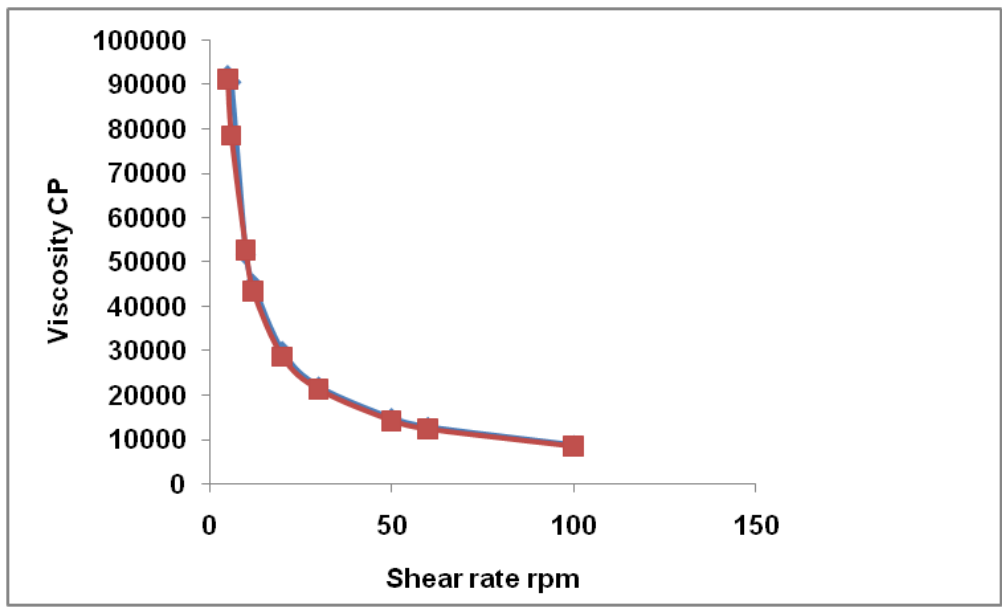

Fig. 3: The rheological profile of combined xanthan-chitosan based gel, results are expressed as mean, $n=3 \pm S D$

\section{Stability studies}

Emulgels were found to be yellow viscous creamy preparation with the smooth homogenous appearance which is similar to the day on which it was formulated.

$\mathrm{PH}$ and the drug release of the formulation were found to be stable under storage for $6 \mathrm{mo}$ at $40^{\circ} \mathrm{C} / 75 \% \mathrm{RH}$ and $4^{\circ} \mathrm{C}$.

\section{Statistical analysis}

The data were analyzed by one-way analysis of variance (ANOVA) at the level of $(\mathrm{P}<0.05)$.

\section{CONCLUSION}

The developed emulgel formula of piroxicam was obtained from using both xanthan gum and chitosan. The combination of xanthan gum and chitosan exhibit optimum release, rheology and stability.

\section{ACKNOWLEDGMENT}

The authors acknowledge, with the thanks pharmaceutics department of Baghdad University for technical and financial support.

\section{AUTHORS CONTRIBUTIONS}

All the authors have contributed equally. 


\section{CONFLICT OF INTERESTS}

Declared none

\section{REFERENCES}

1. Ashara K, Shah K, Soniwala M. Emulgel: a novel drug delivery system. J Pakistan Association Dermatol 2016;26:244-9.

2. Meenakshi D. Emulgel A novel approach to topical drug delivery. Int J Pharma Bio Sci 2013;4:847-56.

3. Latha S, Sridevi G. Role of polymers as gelling agents in the formulation of emulgels. Polymer Sci 2016;2:1.

4. Singh B, Kumar S, Shafaat K. Development and characterization of a nanoemulsion gel formulation for transdermal delivery of carvedilol. Int J Drug Dev Res 2012;4:151-61.

5. Kristl J, Smid-Korbar J, Struc E, Schara MV, Rupprecht H. Hydrocolloids and goals of chitosan as drug carriers. Int J Pharm 1993;99:13-9.
6. Abd-Allah F, Dawaba HM, Ahmed AMS. Preparation characterization, and stability studies of piroxicam loaded microemulsions in topical formulations. Drug Discoveries Ther 2010;4:267-75.

7. Maheswaran A, Padmavathy J, Nandhini V, Saravanan D, Angel P. Formulation and evaluation of floating oral in situ gel of diltiazem hydrochloride. Int J Appl Pharm 2016;9:50.

8. Kini AG, Dixit M, Kulkarni PK. Enhancement of solubility and dissolution rate of poorly water-soluble drug by spray drying using different grades of chitosan. Int J Pharm Pharm Sci 2011;3:231-5.

9. Nur A, Mohamed N, Yagoub N. Comparative evaluation of xanthan, guar and treated guar gums as drug release barriers in oral matrices. Int J Pharm Pharm Sci 2015;7:436-40.

10. Dasgupta S, Mazumder B, Ghosh S, Kaurav S. Solid lipid nanoparticles (SLNs) for topical delivery of aceclofenac by using xanthan gum: ex vivo and in vivo evaluation. Curr Drug Delivery 2013;10:11.

11. Rao M, Sukre G, Aghav S, Kumar M. Optimization of metronidazole emulgel. J Pharm 2013;1:1 